\title{
Effect of Superhydrophobic/Hydrophilic Surfaces on Dynamic Radius of Water Droplet Impact
}

\author{
Masaki Yamaguchi, ${ }^{*}$ Shunsuke Tamura, and Tetsuhiro Sakata \\ Department of Mechanical Engineering and Robotics, Graduate School of Science and Technology, \\ Shinshu University, 3-15-1 Tokida, Ueda, Nagano 386-8567, Japan
}

(Received March 22, 2018; accepted May 15, 2018)

Keywords: droplet impact, dynamic contact angle, interfacial tension, numerical analysis, superhydrophobic surface

In this research, we aim to investigate the dynamic contact angle between water droplets and three different surfaces from the hydrophilic $\left(<90^{\circ}\right)$ to the superhydrophobic region $\left(\cong 150^{\circ}\right.$ in ambient air. The dynamic contact angle and the triple line velocity were experimentally determined from a two-dimensional projection recorded with a high-speed camera in a backlighting setup. The findings from the experiment were used to determine the model parameters of an empirical dynamic contact angle model, which was subsequently used in three-dimensional (3D) numerical simulations. The focus of this work is on the advancing and receding contact angles over the triple line velocity, which is approximated by the model used. The behaviors on the different surfaces were analyzed in detail using the capillary number, and different regimes were identified. It was demonstrated that with the adjusted model, the simulation results regarding the droplet diameter were improved compared with the simulations using static (equilibrium) contact angle models. Thus, it was clarified that the contact angle at the reversal point should be taken and not the static contact angle. Additionally, it was concluded that only one contact angle is needed for a super-hydrophobic system.

\section{Introduction}

The rapid dynamics of the impact process have been studied with the aim of unraveling the underlying physics, which are characterized by the delicate interplay of liquid inertia, viscosity, and surface tension. ${ }^{(1)}$ Droplet impact has many practical applications such as inkjet printing, ${ }^{(2)}$ forensic science, ${ }^{(3)}$ and bioanalysis in the medical field. ${ }^{(4)}$ In biosensors, surface modification by applying a surfactant or a hydrophobic coating agent is common and effective method to prevent non-specific binding. Thus, improvement of hydrophilicity or hydrophobicity by a physical approach can increase the biosensor's sensitivity and selectivity. ${ }^{(5,6)}$

When no splashing is observed, a droplet on a dry surface simply spreads over the surface until it reaches a maximum radius. Thus, an important outcome of a droplet impact on a solid substrate is the maximum spreading droplet radius. The dynamics are then controlled by subtle

*Corresponding author: e-mail: masakiy@shinshu-u.ac.jp

http://dx.doi.org/10.18494/SAM.2018.1944 
balances among the inertia, viscosity, and capillary forces. ${ }^{(7)}$

A number of studies on the numerical simulations of droplet impact based on dynamic contact angles have conducted. ${ }^{(8)}$ Unfortunately, however, there is no fundamental equation for the interfacial tension. The dynamics have generally been considered to be a function of the triple-line velocity. Empirical dynamic contact angle models can be categorized as follows:

(i) models based on a step function using advancing and receding angles, which work well for situations in which inertia dominates, ${ }^{(9,10)}$

(ii) experimental models that work well when capillary flow is dominant, ${ }^{(11,12)}$ and

(iii) models based on Hoffman's experiments in glass capillary tubes for a wide range of capillary numbers. ${ }^{(13,14)}$

Several studies have shown the importance of the velocity of the contact line. A new algorithm for modeling the dynamic contact angle was proposed, in which the dynamic contact angle is a function of the instantaneous velocity of the contact line. ${ }^{(15)}$ To follow the droplet spreading over time, a high-speed video camera was used, allowing the precise determination of the expansion of the droplet and the profile of the free surface at the contact line. ${ }^{(16)}$ Elsewhere, a dynamic contact angle model based on a step function has been modified and used as a semiempirical equation. ${ }^{(17,18)}$ This model expresses the relationship between the dynamic contact angle and the contact line velocity, and has the advantage that it can quantitatively reproduce droplet impact behavior from the impact to the steady state, including the spread and the recoil. Several different impact conditions were analyzed, and it was shown that droplets spread on hydrophilic and superhydrophobic surfaces with different time scales. ${ }^{(19)}$ There is evidence that a droplet can rebound from a surface when the wettability is low. ${ }^{(20)}$

However, the dynamic contact angle is not well understood and is also difficult to measure. Experimentally, Bartolo et al. revealed the influence of different liquid viscosities on the dynamic contact angle using the Ohnesorge number. ${ }^{(7)}$ Another important factor in this class of empirical models is the role played by the material of the solid substrate. Bayer and Megaridis presented guidelines for numerical and analytical studies, according to which careful attention must be paid to the implementation of boundary conditions for the moving contact line. ${ }^{(21)}$ It remains unclear whether it is possible to precisely simulate the water droplet impact without considering the dynamic contact angle. Additionally, the method of determining the model parameters of the empirical model is not sufficiently understood.

In this research, we aim to investigate the dynamic contact angle between water droplets and three different surfaces from the hydrophilic $\left(<90^{\circ}\right)$ to the superhydrophobic region $\left(\cong 150^{\circ}\right)$ in ambient air. The focus of this work is on the advancing and receding contact angles over the triple-line velocity, which is approximated by the empirical dynamic contact angle model used. Firstly, the relationship between the dynamic contact angle and the contact line velocity was measured for each material. A 3D numerical analysis of water droplet impact based on the dynamic contact angle model was then performed. The dynamic droplet radius was evaluated both by numerical analysis and experiment. 


\section{Materials and Methods}

\subsection{Materials}

Chemical surface modification is not the most suitable method to control the equilibrium contact angle because the molecular orientation has a nonlinear effect on the dynamic contact angle. Therefore, to verify the influence of the interfacial tension, three different materials, namely, $\mathrm{SiO}_{2}$ (4 inch $\mathrm{Si}$ wafer with a native oxide, Electronics and Materials Co. Ltd., Japan), $\mathrm{Ag}$, and polytetrafluoroethylene (PTFE, Chukoh Chemical Industries Ltd., Japan), which have different equilibrium contact angles, were used as the solid substrates. The equilibrium contact angles of these materials were measured using a commercial contact angle analyzer (DM-701, Kyowa Interface Science Co. Ltd., Japan) and $1 \mu \mathrm{L}$ of distilled water dropped onto the surfaces. The measurements were repeated 5 times in different areas. The mean values of the measurements were defined as the equilibrium contact angles. The measured equilibrium contact angles $\theta_{e}$ of $\mathrm{SiO}_{2}, \mathrm{Ag}$, and PTFE were $62.9,102.8$, and $149.1^{\circ}$, respectively.

Also, the arithmetic average roughness $\mathrm{Ra}$ of the three materials was measured using a surface roughness meter (Surfcom 480A-12, Tokyo Seimitsu Co. Ltd., Japan) because it is well

known that roughness plays an important role in water droplet impact. ${ }^{(22)}$ The roughnesses of $\mathrm{SiO}_{2}, \mathrm{Ag}$, and PTFE were $<0.01,0.02$, and $0.22 \mu \mathrm{m}$, respectively.

\subsection{Experiment of water droplet impact}

Figure 1 shows the measurement system for water droplet impact based on a high-speed camera. This system consisted of a digital high-speed camera ( $\alpha$ : MEMERCAM HX-6, Nac Image Technology Inc., Japan), an $x y z$-stage ( $\beta$ : TSD-805s, Sigmakoki Co. Ltd., Japan), a static eliminator ( $\gamma$ : SJ-M400, Keyence Co. Ltd., Japan), a noncontact-type capacitive voltmeter ( $\delta$ : SK-035/200, Keyence Co. Ltd., Japan), a $10 \mu \mathrm{L}$ syringe with a 32G needle ( $\zeta:$ Kyowa Interface Science Co. Ltd., Japan), and a light-emitting diode (LED)-type light source ( $\varepsilon$ : LA-HDF5010, Hayashi Watch-Works Co. Ltd., Japan). Apart from the high-speed camera, all the other components of the system were placed in a windproof case $1\left[(\mathrm{~W}) \times 0.5(L) \times 0.75(H) \mathrm{m}^{3}\right]$ to prevent the influence of dust and wind. The time resolution of the high-speed camera was in the range between $20 \mathrm{~ms} /$ frame and $1.54 \mu \mathrm{s} /$ frame [equivalent to 50 and 650000 frames per second (fps)]. The maximum spatial resolution of the high-speed camera was $2560 \times 1920$ pixels $(x \times y$ axis).

Time-dependent images of water droplet impact were recorded using the high-speed camera. There is a trade-off between the time resolution and the spatial resolution. In order to realize a time resolution of $10 \mu \mathrm{s} /$ frame (100000 fps), the number of pixels was set to $512 \times 72(36864$ pixels in total), while the spatial resolution was set to $10 \times 10 \mu \mathrm{m}(x \times y$ axis). As a result, the distance between the high-speed camera and the droplet was $250 \mathrm{~mm}$.

Prior to the experiment, the solid substrates were cleaned in distilled water using an ultrasonic cleaning machine (1510J-MT, Emerson Japan Ltd., Japan) for $10 \mathrm{~min}$. After that, the remaining distilled water on the surface of the solid substrates was dried with nitrogen gas. 


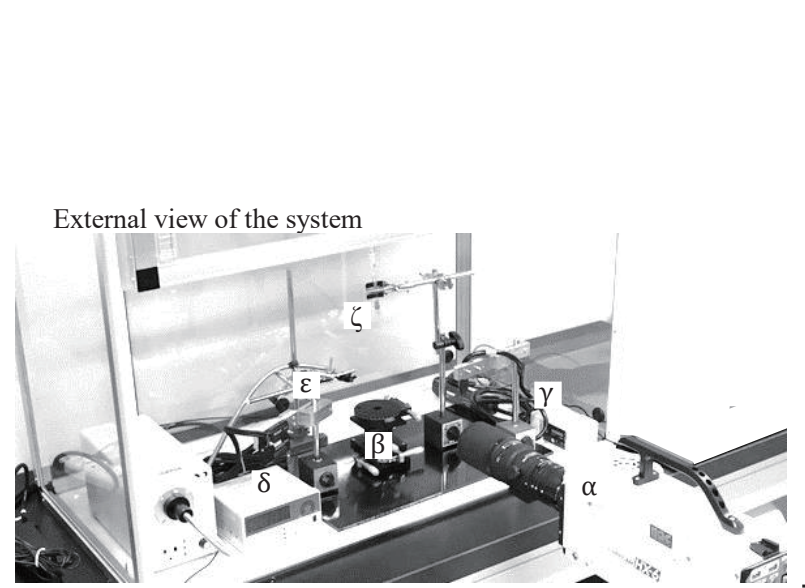

$(\alpha$ : Digital high-speed camera, $\beta$ : $x y z$-stage, $\gamma$ : Static eliminator, $\delta$ : Capacitive voltmeter, $\zeta$ : Syringe, $\varepsilon$ : Light source.)

(a)

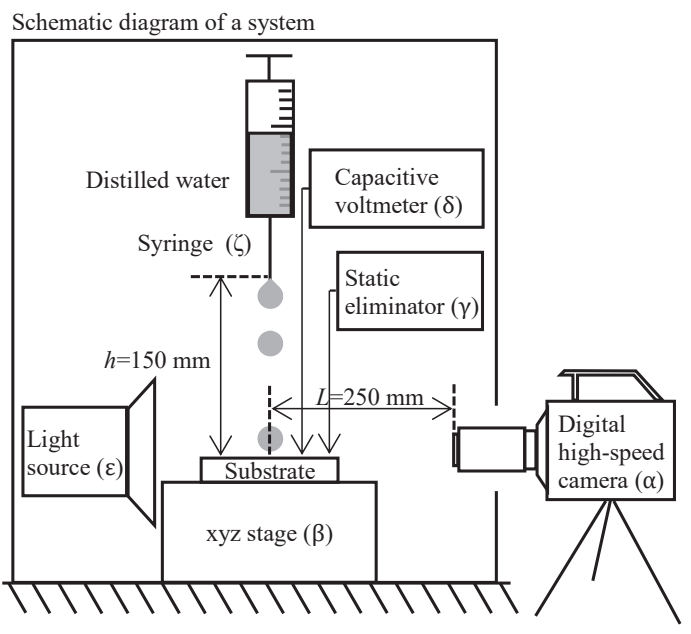

(b)

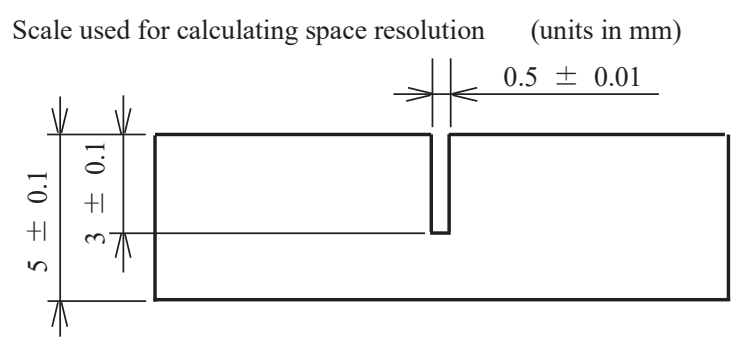

(c)

Fig. 1. Measurement system of a droplet impact based on a high-speed camera.

The solid substrate was placed in a horizontal position on the $x y z$-stage, and then neutralized with the static eliminator. Immediately after this, it was confirmed that the surface voltage had decreased to $\pm 5 \mathrm{~V}$ using the noncontact-type capacitive voltmeter. A $4.6 \mu \mathrm{L}$ droplet of distilled water was dropped onto the solid substrate from a height of $h=150 \mathrm{~mm}$ using the syringe. The water droplet was illuminated from the opposite side to the high-speed camera using the LED-type light source so as to clearly outline the shape of the water droplet. Here, direct light from the light source was blocked by paper to prevent it reaching the camera. A stainless steel scale was fabricated and placed next to the water droplet. The width of groove at $0.5 \mathrm{~mm}$ was used as a standard to measure the diameter of water droplet accurately [Fig. 1(c)]. The room temperature was maintained at $25^{\circ} \mathrm{C}$ throughout the experiment.

Image analysis software (FAMAS, Kyowa Interface Science Co. Ltd., Japan) was used to determine the outline of the water droplet from the backlighting image. As postprocessing of the recorded pictures, the tangent method was used to calculate the dynamic contact angle at the point of the triple line. ${ }^{(23)}$ Two sets of experimental data were collected, one for the relationship between the dynamic contact angle and the contact line velocity, and the other for the timecourse changes of the dynamic radius of the water droplet. 


\subsection{D numerical analysis of water droplet impact}

Thermo-fluid software (scSTREAM Ver.13, Software Cradle Co., Ltd., Japan) based on the continuum surface force (CSF) model using the finite volume method (FVM) and an orthogonal grid was used for the 3D computational fluid dynamics of the water droplet impact. The thermo-fluid software was operated on a parallel computer with 16 degrees of parallelism (DOP). A workstation (Precision T7810, CPU: Dual Intel ${ }^{\circledR}$ Xeon processor E5-2667 $\times 2$ systems, 3.2 GHz, OS: Windows 7, HPC Solutions, Inc., Japan) was used for the calculations.

A Multi-interface Advection and Reconstruction Solver (MARS) was used for interface capturing, ${ }^{(24,25)}$ and a first-order upwind difference scheme was used for calculating the convection term. ${ }^{(26,27)}$ The second-order central difference approximation was used for calculating the viscosity term, and a semi-implicit method for pressure-linked equations was used to calculate the pressure gradient. ${ }^{(28)}$

Figure 2 shows a deformed 3D mesh model overlaid with a rectangular mesh for the numerical analysis (6823604 elements and 6933750 nodes). A quarter-sized model was created on rectangular coordinates in the $x-y$ plane. In the $z$-direction, a grid layer was added to model the solid substrate with a thickness of $12.5 \mu \mathrm{m}$ from the origin. A no-slip boundary condition was applied to the solid-fluid boundary. Also, at the surface of the solid substrate $(x-$ $y$ plane, $z=12.5 \mu \mathrm{m}$ ), a no-slip boundary condition was applied in the immediate vicinity of the moving triple line. No singularity was produced in this numerical analysis because there was no calculation point on the border between no-slip and free-slip. From the origin to $1 / 2$ of the droplet radius, the grid widths were set to $12.5 \mu \mathrm{m}$, in order to accurately model the important behavior on the solid substrate. The time step and courant number were set to be $1.0 \mu$ s and 0.1 , respectively. The density, viscosity, and surface tension of water were set to $997.04 \mathrm{~kg} / \mathrm{m}^{3}, 0.8899$ $\mathrm{mPa} \cdot \mathrm{s}$, and $71.972 \mathrm{mN} / \mathrm{m}$, respectively. The density and viscosity of air were set to $1.19 \mathrm{~kg} / \mathrm{m}^{3}$ and $0.0189 \mathrm{mPa} \cdot \mathrm{s}$, respectively.

The dynamic contact angle $\theta_{v}$ is given by the dynamic contact angle model (Fig. 3) using the equilibrium contact angles $\theta_{e}$ as follows: ${ }^{(17,18)}$

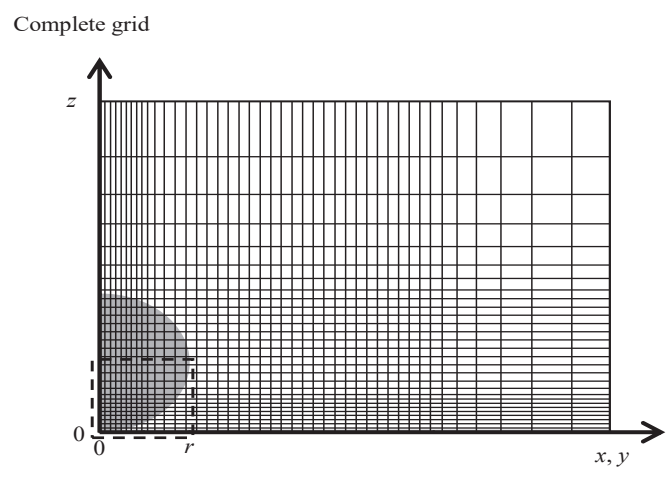

(a)

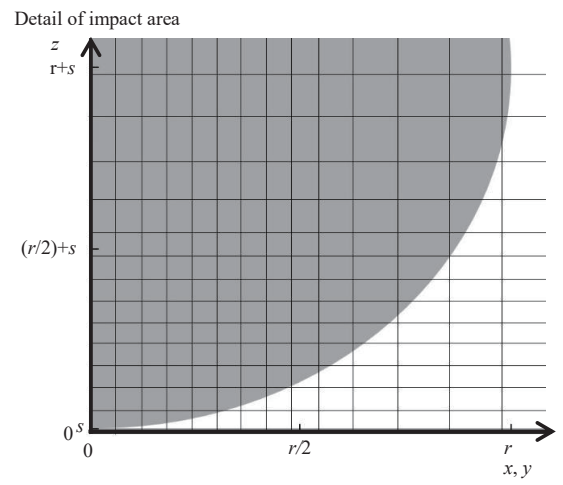

(b)

Fig. 2. Deformed 3D mesh model overlaid with a rectangular mesh ( $r$ : droplet radius of $1.03 \mathrm{~mm}, s$ : grid layer of the solid substrate for a thickness of $12.5 \mu \mathrm{m}$ ). 


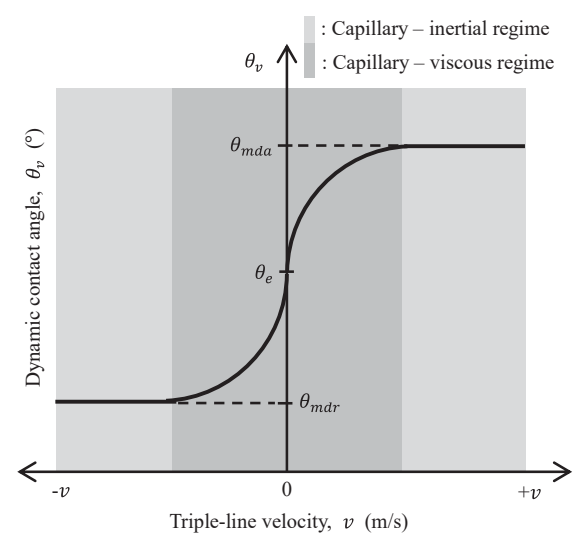

Fig. 3. Relationship between the dynamic contact angle and the triple line velocity given by the dynamic contact angle model.

$$
\theta_{v}=\left\{\begin{array}{l}
\operatorname{Min}\left[\theta_{e}+\left(\frac{C a}{k_{a}}\right)^{1 / 3}, \theta_{m d a}\right] \text { if } v \geq 0 \\
\operatorname{Max}\left[\theta_{e}+\left(\frac{C a}{k_{r}}\right)^{1 / 3}, \theta_{m d r}\right] \text { if } v<0
\end{array}\right.
$$

where $\mathrm{Ca}$ : capillary number,

$k_{a} \quad:$ material-related parameter for the advancing situation,

$k_{r} \quad:$ material-related parameter for the receding situation,

$\theta_{m d a} \quad$ : maximum dynamic advancing angle $\left({ }^{\circ}\right)$,

$\theta_{m d r} \quad$ : minimum dynamic receding angle $\left(^{\circ}\right)$,

$v \quad:$ triple-line velocity $(\mathrm{m} / \mathrm{s})$.

In the capillary-inertial force regime, where $\mathrm{Ca}$ is relatively high, the model employs the constant angles $\theta_{m d a}$ and $\theta_{m d r}$ for the advancing and receding situations, respectively. ${ }^{(7)}$ In the capillary-viscous force regime, where $\mathrm{Ca}$ is relatively low, the model uses empirical equations. The parameters $k_{\mathrm{a}}$ and $k_{\mathrm{r}}$ are determined as the best fit parameters to the measured results of the relationship between the dynamic contact angle and the contact line velocity.

\section{Results and Discussion}

\subsection{Experiment of water droplet impact}

Figure 4 shows images of water droplet impact using $\mathrm{SiO}_{2}, \mathrm{Ag}$, and PTFE as the solid substrate. The volume of the distilled water droplet, determined from its outline, was $4.6 \mu \mathrm{L}$. The impact velocity ( $t=0 \mathrm{~s}$ ) of the water droplet was estimated to be $1.67 \mathrm{~m} / \mathrm{s}$ from the images. For each of the three materials, the water droplets falling onto the dry surfaces simply spread over the surface until they reached a maximum radius, while no splashing was observed. The water droplet became pancakelike in shape, for example as shown in Fig. 4(a) after 3.0 ms when $\mathrm{SiO}_{2}$ was used as the solid substrate (the triple-line velocity of $0.05 \mathrm{~m} / \mathrm{s}$ ).

Figure 5 shows the experimental relationship between the dynamic contact angle and the 


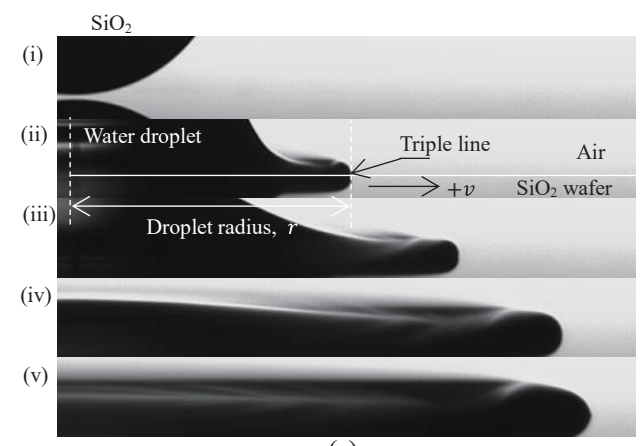

(a)

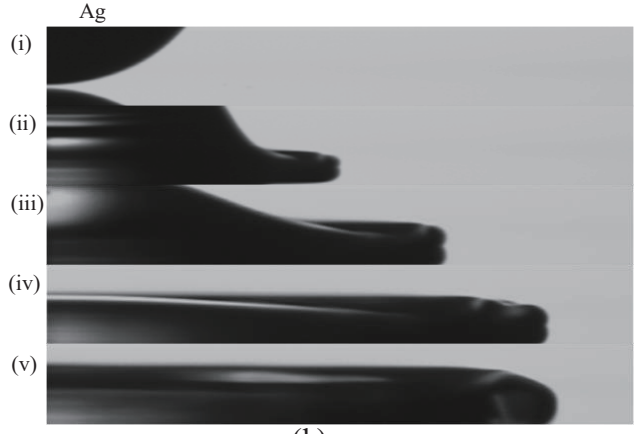

(b)

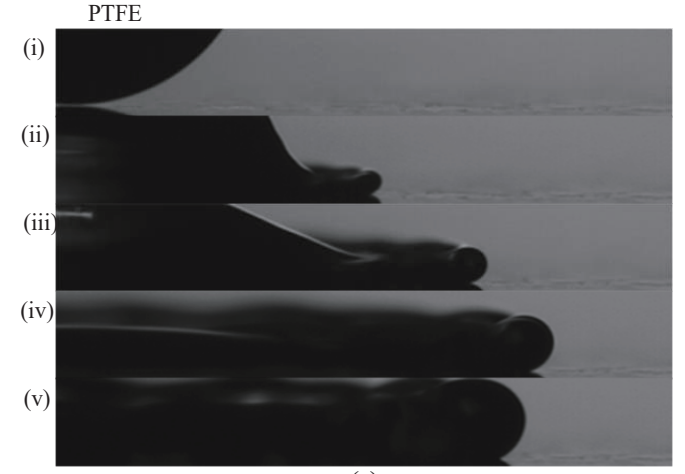

(c)

Fig. 4. Images of the water droplet impact of each solid substrate using a $4.6 \mu \mathrm{L}$ droplet of distilled water at intervals: (i) $0.0 \mathrm{~ms}$, (ii) $0.5 \mathrm{~ms}$, (iii) $1.0 \mathrm{~ms}$, (iv) $2.0 \mathrm{~ms}$, and (v) $3.0 \mathrm{~ms}$.

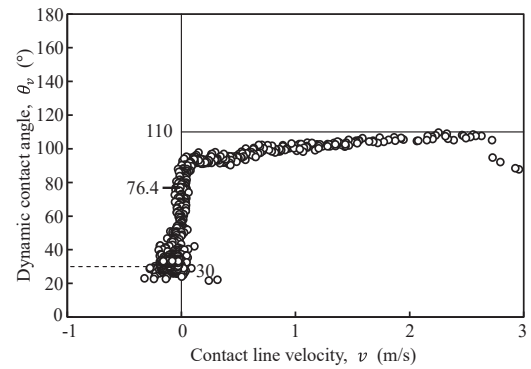

(a)

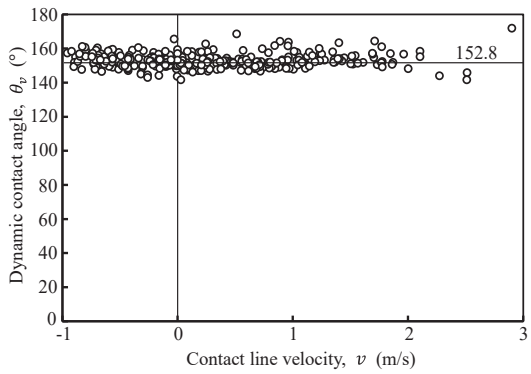

(c)

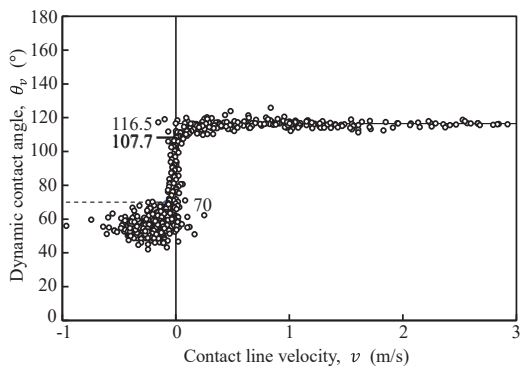

(b)

Fig. 5. Experimental results for the relationship between the dynamic contact angle and the contact line velocity using a $4.6 \mu \mathrm{L}$ droplet of distilled water. (a) $\mathrm{SiO}_{2}$, (b) $\mathrm{Ag}$, and (3) PTFE. 
contact line velocity using a $4.6 \mu \mathrm{L}$ droplet of distilled water. For droplet impact on the PTFE solid substrate, the dynamic contact angle was constant in both plus and minuses contact line velocities, with a mean of $152.8^{\circ}$. This results has a meaning that there was no deformation of water droplet at the triple line for PTFE after impact. The PTFE substrate evidently did not fall into the capillary-viscous regime, which was attributed to the high equilibrium contact angle of PTFE, putting it in the superhydrophobic region.

The dynamic contact angles at a contact line velocity of $0 \mathrm{~m} / \mathrm{s}$, denoted by $\theta_{v}=0$, for $\mathrm{SiO}_{2}$ and $\mathrm{Ag}$ were 76.4 and $107.7^{\circ}$, respectively. These measurements were performed under the conditions of controlling the surface roughness of the solid substrates, the dust and wind, and the static electricity. The $\theta_{v}=0$ values did not agree with the equilibrium contact angles $\left(\theta_{e}\right.$ $=62.9$ and $102.8^{\circ}$ ). These measured equilibrium contact angles of $\mathrm{SiO}_{2}$ and $\mathrm{Ag}$ did, however, agree reasonably well with those in a previous report. ${ }^{(29)}$ The discrepancy between the dynamic and equilibrium contact angles is to be expected, considering that the geometries of the water droplets changed over time, being spherical at $\theta_{e}$ and pancakelike at $\theta_{v}=0$. Therefore, in the dynamic contact angle model, the values of $\theta_{v}=0$ should be used in place of $\theta_{e}$. From the absolute values of the equilibrium contact angle, it was considered that the $\mathrm{SiO}_{2}$ and $\mathrm{Ag}$ surfaces were in an advanced state of oxidation.

Using these experimental results, $k_{a}, k_{r}, \theta_{m d a}$, and $\theta_{m d r}$ for the $\mathrm{SiO}_{2}$ solid substrate were determined to be $8.2 \times 10^{-7}, 2.0 \times 10^{-8}, 110^{\circ}$, and $30^{\circ}$, respectively. $k_{a}$ and $k_{r}$ were obtained from approximate curves using Eq. (1). For $\mathrm{SiO}_{2}$, the values of $\theta_{m d a}$ were set to the asymptotic values, while those of $\theta_{m d r}$ were set to the mean values between -0.1 and $-0.2 \mathrm{~m} / \mathrm{s}$. The corresponding values for the Ag solid substrate were determined to be $3.3 \times 10^{-5}, 2.0 \times 10^{-8}$, $116.5^{\circ}$, and $70^{\circ}$, respectively. For droplet impact on the Ag solid substrate, the experimental results of $\theta_{m d r}$ did not show a stable value, and fluctuated between 70.3 and $42.2^{\circ}$, which was attributed to the pinning effect of the surface roughness. It was considered that the maximum value of the measurements should be used $\theta_{m d a}$.

Figure 6 shows the experimental time-course changes of the dynamic radius of the water droplet for each solid substrate using a $4.6 \mu \mathrm{L}$ droplet of distilled water. For PTFE, it was difficult to obtain stable data beyond $4 \mathrm{~ms}$ because the edges of the droplets oscillated. The maximum dynamic radii on $\mathrm{SiO}_{2}, \mathrm{Ag}$, and PTFE were 3.27, 3.14, and $2.67 \mathrm{~mm}$, respectively. The times taken for the radii to reach their maximum values are indicated by the dotted lines. These were 3.45, 2.60, and $1.90 \mathrm{~ms}$ for $\mathrm{SiO}_{2}, \mathrm{Ag}$, and PTFE, respectively. The maximum dynamic radius and the arrival time both decreased as the equilibrium contact angle increased. This is consistent with the fact that the capillary force resulting from interfacial tension decreases with increasing equilibrium contact angle.

\subsection{Numerical analysis of water droplet dynamics}

The impact velocity $(t=0 \mathrm{~s})$ of the water droplet was set to $1.67 \mathrm{~m} / \mathrm{s}$, which was equivalent to the estimated value in the experiment. The surface thickness of the solid substrate was predefined as $12.5 \mu \mathrm{m}$, requiring 7 million elements to be generated. For each model, between 12 and $28 \mathrm{~h}$ of real time was needed to calculate the results.

Figure 7 presents experimental and numerical droplet shapes of $\mathrm{SiO}_{2}$ at various times. In 


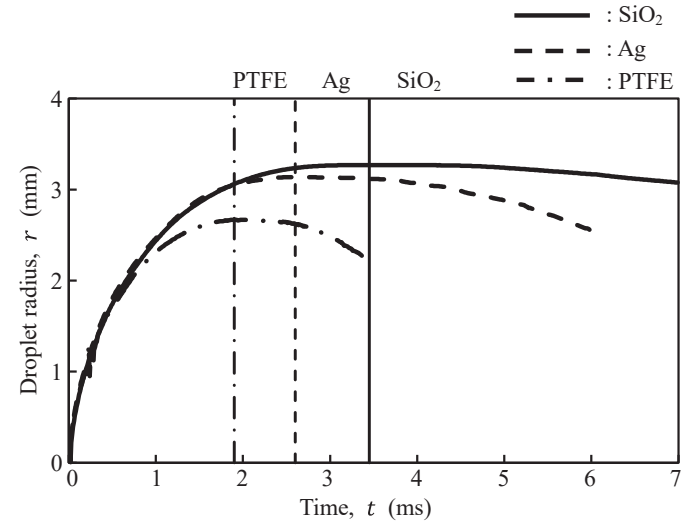

Fig. 6. Experimental results for the time-course changes of the dynamic radius of a water droplet for each solid substrate using a $4.6 \mu \mathrm{L}$ droplet of distilled water.

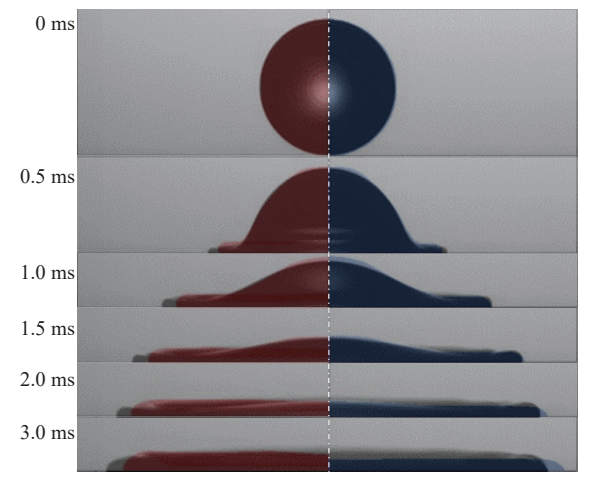

Fig. 7. (Color) Numerical simulations with model (left half, red), numerical simulations without model (right half, blue), and experimental (black) results of $\mathrm{SiO}_{2}$ solid substrate until the dynamic radii reached around the maximum value using a $4.6 \mu \mathrm{L}$ droplet of distilled water.

visually, remarkable difference was not observed in the agreements of the shapes between numerical simulations and experiments in both conditions until $1.0 \mathrm{~ms}$. Some minor differences can be recognized between numerical simulations and experiments in both conditions, which were attributed to the initial oscillations of the droplets before impact, which leads to slightly non-axisymmetric shapes. After that, the agreements of the shapes between numerical simulations with model (left half, red) and experiments are quit good; especially at $3.0 \mathrm{~ms}$.

Figure 8 compares the time-course changes of the dynamic droplet radius between the experimental and calculated results for each solid substrate using a $4.6 \mu$ Ldroplet of distilled water. The calculation results were obtained from $0 \mathrm{~s}$ to twice the time needed to reach the maximum dynamic radius. The capillary numbers obtained from the measured values of $\mathrm{SiO}_{2}$, $\mathrm{Ag}$, and PTFE ranged between 0 and 0.026. For $\mathrm{SiO}_{2}$ [Fig. 7(a)], the radii calculated without the dynamic contact angle model were higher than the measured values, while the radii calculated with the dynamic contact angle model were lower than the measured values. This is consistent with the fact that the capillary force was calculated to be smaller when the dynamic contact angle model was not applied.

To evaluate the agreement between the measured and calculated results, the residual sum of squares (RSS) was calculated by normalizing with the maximum dynamic radius (Table 1). For $\mathrm{SiO}_{2}$ and $\mathrm{Ag}$, the RSS was smaller when the dynamic contact angle model was applied than when it was not applied. In fact, without the model, the RSS for both materials was $\sim 1.8$ times larger than the $\pm 5 \%$ error. It was considered that grid widths of $12.5 \mu \mathrm{m}$ were sufficient to achieve calculation precision with the first-order upwind difference scheme. It was concluded that for materials dominated by the capillary force resulting from interfacial tension, including commonly used materials such as silicon and metals, the dynamic contact angle model significantly improves the simulation accuracy of the water droplet impact. For PTFE, the RSS calculated without the dynamic contact angle model was less than the $\pm 5 \%$ error, indicating that even without the model, the calculations agreed well with the experiments for 


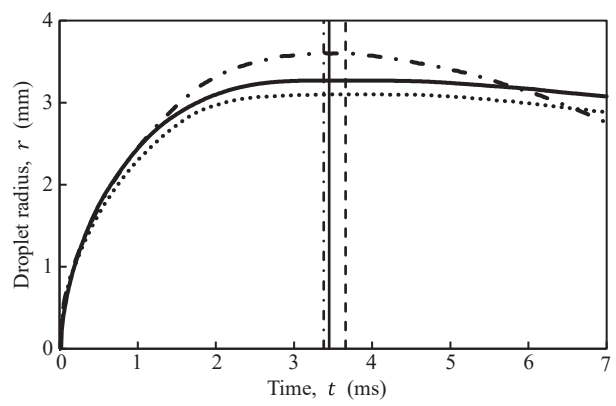

(a)

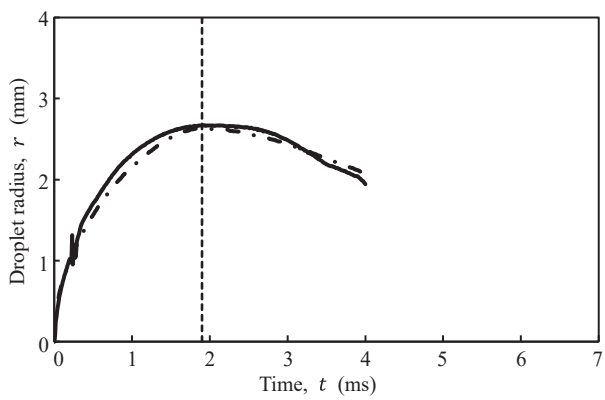

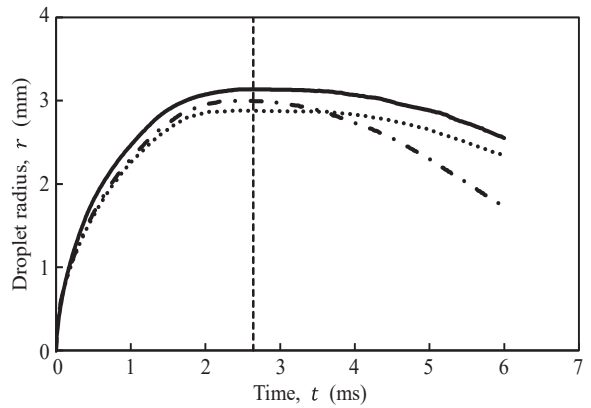

(b)

(c)

Fig. 8. Comparison of the time-course changes of the dynamic droplet radius between the experimental and the calculated results for each substrate. (a) $\mathrm{SiO}_{2}$, (b) Ag, and (3) PTFE.

Table 1

Residual sum of squares between the measured and the calculated results.

\begin{tabular}{lccc}
\hline Solid substrate & With model & Without model & $\pm 5 \%$ error \\
\hline $\mathrm{SiO}_{2}$ & 0.017 & 2.620 & 1.427 \\
$\mathrm{Ag}$ & 0.018 & 1.877 & 1.029 \\
$\mathrm{PTFE}$ & - & 0.242 & 0.689 \\
\hline
\end{tabular}

this superhydrophobic surface $\left(\theta_{e}=149.1^{\circ}\right)$. It was concluded that in a domain where the force is dominated by the viscosity, such as on a super-hydrophobic surface, it is not always necessary to consider the dynamic contact angle when simulating the water droplet impact in cases where this angle remains constant.

The calculation error in each case was calculated at the point in time when the measured dynamic radius had reached its maximum value (Table 2). For the hydrophilic surface $\left(\theta_{e}=\right.$ $62.9^{\circ}$ ) of $\mathrm{SiO}_{2}$, a smaller error was obtained when the dynamic contact angle model was applied than when it was not applied. With the increase in $\theta_{\mathrm{e}}$ to $102.8^{\circ}$, the calculation error with the dynamic contact angle model slightly increased from 5.24\% (for $\mathrm{SiO}_{2}$ ) to $8.27 \%$ (for $\mathrm{Ag}$ ). This increase probably reflected the influence of the interfacial tension of the solid substrates. 
Table 2

Comparison of calculation error based on the measured results.

\begin{tabular}{lccc}
\hline \multirow{2}{*}{ Solid substrates } & $\begin{array}{c}\text { Water droplet radius* } \\
(\mathrm{mm})\end{array}$ & $\begin{array}{c}\text { Calculation error } \\
(\%)\end{array}$ \\
\hline \multirow{2}{*}{$\mathrm{SiO}_{2}$} & Experiments & 3.27 & - \\
& With model & 3.10 & 5.24 \\
& Without model & 3.60 & 10.03 \\
\hline \multirow{2}{*}{$\mathrm{Ag}$} & Experiments & 3.14 & - \\
& With model & 2.88 & 8.27 \\
& Without model & 3.00 & 4.52 \\
\hline \multirow{2}{*}{ PTFE } & Experiments & 2.67 & - \\
& Without model & 2.63 & 1.50 \\
\hline
\end{tabular}

*The calculation errors were obtained at the time point when the measured droplet radii reached the maximum values.

\section{Conclusions}

The focus of this work is on the advancing and receding contact angles over the triple line velocity, which is approximated by the empirical dynamic contact angle model used. The behaviors on the different surfaces were analyzed in detail using the capillary number, and different regimes were identified. It was demonstrated that with the adjusted model, the simulation results regarding the droplet diameter are improved compared with simulations using static contact angle models. Thus, it was clarified that the contact angle at the reversal point should be taken and not the static contact angle. Where the capillary force resulting from interfacial tension was high, such as for commonly used industrial materials, the measured data agreed well with the calculated data when the dynamic contact angle model was applied. On the other hand, it was concluded that only one contact angle is needed when the capillary force is low, such as for a material with a high equilibrium contact angle in the superhydrophobic system $\left(\cong 150^{\circ}\right)$.

These findings might be useful for numerical simulations of sliding behavior of liquid droplets on flat surface and surface with microperiodic structures. ${ }^{(30)}$

\section{Acknowledgments}

The authors wish to thank Dr. Kensuke Yokoi, Cardiff University, for his helpful advice on the numerical simulations. This work was supported by grant No. 16H03166 from the Japan Society for the Promotion of Science, visualization of cancer by cytokine coding method and biosensor array with microcapsules (P.I.: M. Yamaguchi), Japan.

\section{References}

1 C. Josserand and S. T. Thoroddsen: Annu. Rev. Fluid Mech. 48 (2016) 365.

2 H. Minemawari, T. Yamada, H. Matsui, J. Tsutsumi, S. Haas, R. Chiba, R. Kumai, and T. Hasegawa: Nature 475 (2011) 364.

3 N. Laan, K. G. de Bruin, D. Bartolo, C. Josserand, and D. Bonn: Phys. Rev. Appl. 2 (2014) 044018.

4 E. Ueda and P. A. Levkin: Adv. Mater. 25 (2013) 1234.

5 G. E. Jogia, T. Tronser, A. A. Popova, and P. A. Levkin: Microarrays (Basel). 5 (2016) 28. 
6 F. Guittard and T. Darmanin: Bioinspired Superhydrophobic Surfaces: Advances and Applications with Metallic and Inorganic Materials (Pan Stanford Publishing Pte. Ltd., Singapore, 2018) p. 13.

7 D. Bartolo, C. Josserand, and D. Bonn: J. Fluid Mech. 545 (2005) 329.

8 P. de Gennes, F. Brochard-wyart, and D. Quéré, Eds.: Capillarity and Wetting Phenomena Drops, Bubbles, Pearls, Waves (Springer Science+Business Media, Inc., New York, 2004) p. 230.

9 J. Fukai, Z. Zhao, D. Poulikakos, C. M. Megaridis, and O. Miyatake: Phys. Fluids A 5 (1993) 2588.

10 H. Liu, S. Krishnan, S. Marella, and H. S. Udaykumar: J. Comput. Phys. 210 (2005) 32.

11 L. M. Hocking: Proc. 2nd Int. Colloquium on Drops and Bubbles (1982) Paper No. JPL-NASA 82-7, 315.

12 P. D. M. Spelt: J. Comput. Phys. 207 (2005) 389.

13 R. L. Hoffman: J. Colloid Interface Sci. 50 (1975) 228.

14 S. F. Kistler, J. C. Berg, Ed.: Hydrodynamics of Wetting (Dekker, New York, 1993) p. 311.

15 I. V. Roisman, L. Opfer, C. Tropea, M. Raessi, J. Mostaghimi, and S. Chandra: Colloids Surf. A 322 (2008) 183.

16 D. C. Vadillo, A. Soucemarianadin, C. Delattre, and D. C. D. Roux: Phys. Fluids 21 (2009) 122002.

17 K. Yokoi, D. Vadillo, J. Hinch, and I. Hutchings: Phys. Fluids 21 (2009) 072102.

18 K. Yokoi: Soft Matter 7 (2011) 5120.

19 C. Antonini, A. Amirfazli, and M. Marengo: Phys. Fluids 24 (2012) 102104.

20 C. Antonini, F. Villa, I. Bernagozzi, A. Amirfazli, and M. Marengo: Langmuir 29 (2013) 16045.

21 I. S. Bayer and C. M. Megaridis: J. Fluid Mech. 558 (2006) 415.

22 L. Xu, L. Barcos, and S. R. Nagel: Phys. Rev. E: Stat. Nonlinear Soft Matter Phys. 76 (2007) 066311.

23 R. J. Good and R. R. Stromberg, Eds.: Surface and Colloid Science Vol. II (Plenum Press, New York, 1979) p. 36.

24 J. U. Brackbill, D. B. Kothe, and C. Zemach: J. Comput. Phys. 100 (1992) 335.

25 T. Kunugi: Comput. Fluid Dyn. J. 9 (2000) 1.

26 S. E. Rogers and D. Kwak: Am. Inst. Aeronaut. Astronaut. J. 28 (1990) 253.

27 A. J. Chorin: J. Comput. Phys. 135 (1997) 118.

28 S. V. Patankar, Ed.: Numerical Heat Transfer and Fluid Flow (Hemisphere Publishing Co., New York, 1980) p. 131.

29 R. A. Erb: J. Phys. Chem. 69 (1965) 1306.

30 S. K. Alen1, N. Farhat, and Md. A. Rahman: AIP Conf. Proc. (AIP, 2016) 1754. 